\title{
Electron microscopic and immunohistochemical findings in a case of olfactory neuroblastoma
}

\author{
Z M Du, Y S Li, B F Wang
}

\begin{abstract}
A case of olfactory neuroblastoma is reported. Light microscopic examination showed various arrangements of poorly differentiated tumour cells forming either uniform sheets or convoluted cords of multiple cell layers orientated toward a richly vascular stroma. Electron microscopic examination showed the presence of abundant cytoplasmic filaments and processes, and dense core endocrine vesicles ranging from $100-160 \mathrm{~nm}$ in diameter in both the perinuclear region and tumour cell processes. Immunohistochemical staining was positive in most of the tumour cells for neuron specific enolase, and in a few cells for S-100 protein, vimentin, and serotonin, but staining for desmin and keratin produced no reaction.
\end{abstract}

(F Clin Pathol 1993;46:83-85)

Olfactory neuroblastoma is a rare neoplasm, originally described by Berger in 1924 and 1926 under the names of olfactory aesthesioneurocytoma and aesthesioneuroepithelioma. ${ }^{1}$ It generally arises in the nasal cavity, often above and beside the ethmoid sinus. Its age distribution is bimodal, one peak occurring in adolescence and the other in the sixth decade, although it may be found at any age. The sex incidence is about equal. The histogenesis of this tumour is unknown, though it is suggested that it arises from the neuroepithelial sensory cells of the olfactory mucosa. We describe a case where detailed microscopic assessment using current techniques was possible.

\section{Case report}

A 54 year old man presented with nasal discharge that had persisted for about one month. An uneven surface neoplasm was located in the nasal septum obstructing the right nostril.

A computed tomogram indicated a tumour mass spreading to the right nasal cavity and paranasal sinus. At surgery it was found that the neoplasm had mainly invaded the upper part of nasal cavity; its size was about $3 \times 2 \times$ $2.5 \mathrm{~cm}$. The tumour had infiltrated the ethmoidal sinus and the medial concha and was 1.5 $\mathrm{cm}$ away from the nasopharynx. The sphenoidal and maxillary sinuses were unaffected.
The patient received radiotherapy and responded well. There was no sign of recurrence at the time of writing.

Paraffin wax sections were used for the histological and immunohistochemical studies. Primary antibodies included rabbit antihuman neuron-specific enolase (NSE) with a titre of $1 / 200, S-100$ protein $(1 / 200)$, keratin $(1 / 400)$ antibodies, and monoclonal antibodies to vimentin and desmin (1/200) (Sigma). The serotonin antibody (5-HT) with a titre of $1 / 1000$ was provided by the department of histology at our university. All immunohistochemical staining was performed using the $A B C$ method; the $A B C$ kit was purchased from Victor Company.

Specimens of less than $1 \mathrm{~mm}$ in diameter were routinely processed and ultrathin sections were stained with uranyl acetate and lead citrate for electron microscopy.

\section{Results}

MICROSCOPIC APPEARANCES

In haematoxylin and eosin stained sections, the tumour tissue showed poorly differentiated neuroblasts forming either uniform sheets against a delicately fibrillated background or convoluted cords of multiple cell layers orientated towards a richly vascular stroma (fig 1 ), with a suggestion of whorls or a rossettelike structure. The tumour cells were about 8-12 $\mu \mathrm{m}$ in diameter with round, oval, or kidney-shaped nuclei and eosinophilic cytoplasm. Argyrophilic staining showed abundant neuro fibrils and dendritic processes originating from the tumour cells. Argyrophilic granulates were also present in some cells. Tumour cell nests had infiltrated into the surrounding normal tissue. There was prominent necrosis of some tumour tissue which seemed related to poor blood supply.

\section{ULTRASTRUCTURAL FINDINGS}

The tumour cells contained convoluted nuclei with additional invaginations thus forming multiple nuclear pockets. The cytoplasm was poorly differentiated, except for abundant free ribosomes, with scant organelles (fig 2), although the mitochondria, endoplasmic reticulum, glycogen, lysosomes and autophagosomes were present to some degree. A prominent feature was the presence of dense core vesicles ranging from 100 to $160 \mathrm{~nm}$ in diameter and scattered mainly in the cytoplasmic processes (fig 3), though their number 
Figure 1 Micrograph of olfactory neuroblastoma, showing tumour cells arranged in convoluted whorls around the stromal tissue with abundant blood vessels.

Figure 2 Electron micrograph showing the pleomorphic nucleus forming the pockets, and abundant free ribosomes. lysosomes, and autophagosomes.
Figure 3 Tumour cell showing dense core granules ranging from 100 to $160 \mathrm{~nm}$ in diameter and clear centred vesicles in cytoplasmic processes.
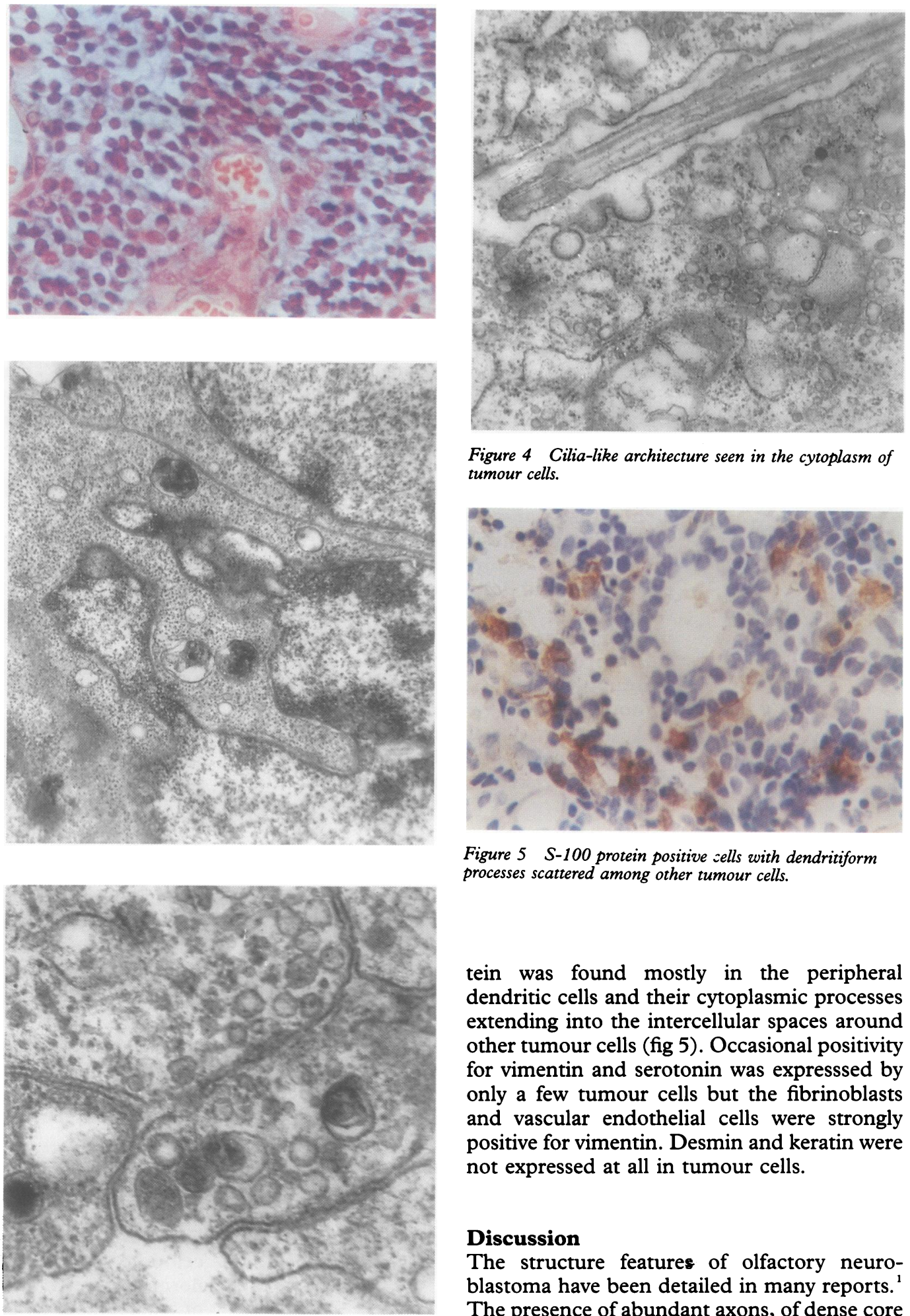

was small. Another characteristic was the presence of abundant cytoplasmic processes, axon-like vesicles, and compact bundles of cytoplasmic filaments, presumably neurofilaments. Ciliated structures, or basal bodies, were also detected in a few tumour cells (fig 4), indicating some differentiation towards mucosal epithelial cells.

IMMUNOHISTOCHEMICAL STAINING

Most of the tumour cells had a moderately positive reaction to the antibody against neuron-specific enolase. Positivity to S-100 pro-
Figure 4 Cilia-like architecture seen in the cytoplasm of tumour cells.

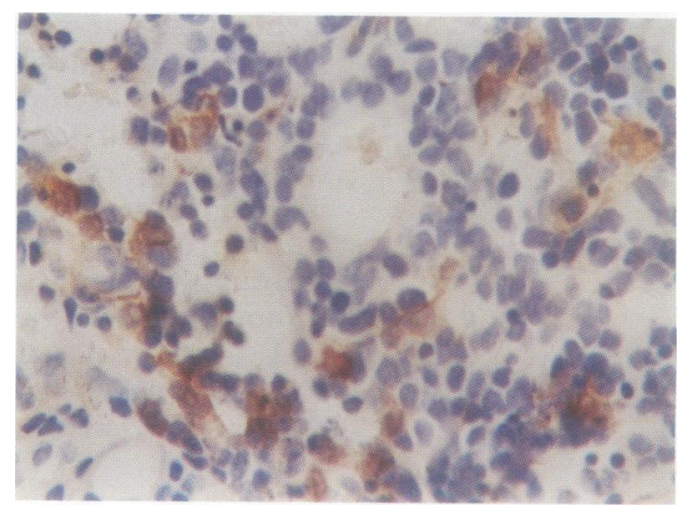

Figure 5 S-100 protein positive sells with dendritiform processes scattered among other tumour cells.

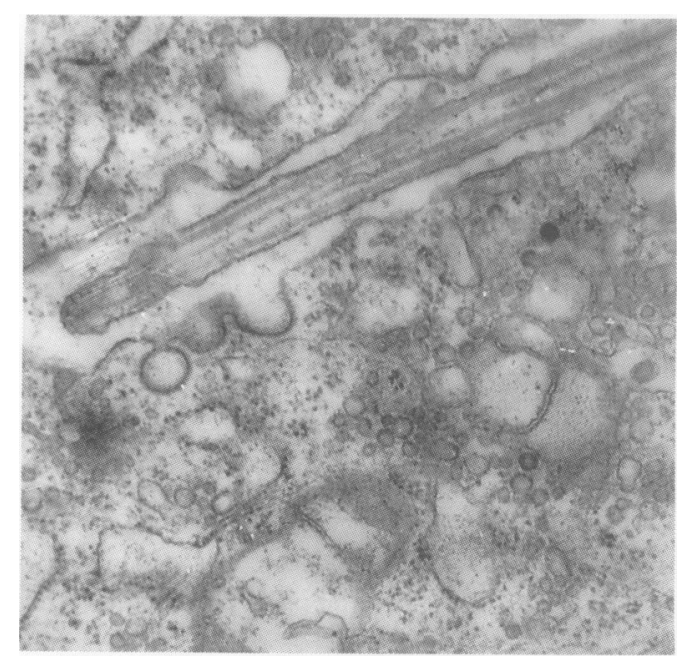

tein was found mostly in the peripheral dendritic cells and their cytoplasmic processes extending into the intercellular spaces around other tumour cells (fig 5). Occasional positivity for vimentin and serotonin was expresssed by only a few tumour cells but the fibrinoblasts and vascular endothelial cells were strongly positive for vimentin. Desmin and keratin were not expressed at all in tumour cells.

\section{Discussion}

The structure features of olfactory neuroblastoma have been detailed in many reports. ${ }^{1}$ The presence of abundant axons, of dense core vesicles of various sizes, numerous cytoplasmic processes, microtubules and synaptic complexes, all offer evidence of the neuronal nature of this neoplasm. The described number and size of the dense core vesicles has varied greatly in previous reports, possibly related to the variable endocrine activity of different tumours.

A separate variant, regarded as an example of true aesthesioneuroepithelioma originating from sensory neurons and sustentacular cells of the olfactory epithelium, and devoid of dense core granules, has also been described. ${ }^{2}$ The dense core granules of tumour cells in our case were comparatively rare, but could be 
clearly demonstrated by electron microscopy, suggesting that the tumour was poorly differentiated. The presence of abundant cytoplasmic processes, clear centred vesicles, and compact bundles of cytoplasmic filaments, presumably neurofilaments, were typical of the described morphological features of olfactory neuroblastoma.

Cilia-like structures and basal bodies have been observed in some tumour cells, suggesting partial differentiation towards olfactory epithelium. Silva $e t a l^{3}$ reported that olfactory epithelium was observed in three of nine cases of olfactory neuroblastoma. The occasional association of olfactory neuroblastoma with adenocarcinoma and even squamous carcinoma has also been described. These exceptional mixed cell cases are regarded as instances of divergent neoplastic differentiation, presumably originating from undifferentiated basal cells. Olfactory eipthelium consists of three cell types, basal cells, supporting cells, and sensory neurons, so it is logical that differentiation of epithelial cells arises in this tumour.

The most consistently positive immunochemical reaction is that for neuron-specific enolase. S-100 protein positive cells are found mostly in peripheral Schwann cells. Axe $e t a l^{4}$ reported that of eight cases of olfactory neuroblastoma, six were positive for NSE, five for SD-100 protein, and one for neuron filament protein. When keratin is expressed, it may be associated with frank squamous differentiation. We observed that most tumour cells were positive for NSE, and some dendritic cells were positive for S-100 protein, which con- firmed the diagnosis of olfactory neuroblastoma.

Vimentin positivity is usually regarded as characteristic of sarcomas, but a positive reaction was found in a few tumour cells in this case. It was demonstrated by Schwob et al that vimentin is the only intermediate protein present in most neurons in the olfactory epithelium, a feature which they share with the early, more undifferentiated stage of normal neuronal development. Vimentin positivity of tumour cells may be the expression of an embryonal antigen.

Silva $e t ~ a l^{3}$ subdivided the tumour into neuroblastoma and neuroendocrine carcinoma, both groups showing different age incidences, recurrence rates, and metastatic potential. The main features of our case are more in accordance with neuroblastoma than neuroendocrine carcinoma, and the observed negative reaction to keratin and desmin suggested no component of adenocarcinoma or squamous carcinoma to be present.

1 Russell DS, Rubinstein IG. Pathology of tumors of the nervous system. 5th edn. London; Edward Arnold, 1989:915-20. 2 Hassoun J, Gambarelli D, Grisoli F, Henric A, Toga M. Ethesioneuroepithelioma, a true neurosensorial tumour. Light and electron microscopic study of a case with Light and electron microscopic study of a case with

3 Silva EG, Butler JJ, Mackay B, Goepfert H. Neuroblastoma and neuroendocrine carcinoma of nasal cavity. A proand neuroendocrine carcinoma of nasal cavity. A

4 Axe S, Kuhajda FP. Esthesioneuroblastoma. Intermediate filaments, neuroendocrine, and tissue-specific antigens. Am 7 Clin Pathol 1987;88:139-45.

5 Schwob JE, Farber NB, Gottlieb DI. Neurons of the olfactory epithelium in adult rats contain vimentin. $\mathcal{f}$ Neurosci 1986;6:208-14. 